\title{
Electroconvulsive therapy and its use in modern-day psychiatry
}

To the Editor: The aim of this article' was stated as 'to give a general overview of the use of ECT and an updated overview of newer research in this field. It also gives practical guidelines for administering ECT.

Unfortunately many of the articles quoted in this review have been overtaken by more up-to-date publications, especially in previously controversial areas, as will be discussed below. The following in particular warrant further discussion.

\section{Bilateral (BL) versus unilateral (UL) ECT}

Prinsloo and Pretorius quote a paper by Sackeim et al. ${ }^{2}$ published in 1993, in which the response rate for 'high-dose' UL ECT was $43 \%$ compared with $63 \%$ for high-dose BL ECT. ${ }^{2}$

Unfortunately Prinsloo and Pretorius do not provide a conclusion based on their review of the literature, and they fail to include any of a number of newer publications in this area. They fail to point out that in this particular study ${ }^{2}$ the principal weakness of the methodology was the fact that the stimulus intensities were only given at a maximum dose of 2.5 times seizure threshold in both the UL and the BLECT group. Indeed, Sackeim et al. ${ }^{2}$ point out that the dose variability in the previously published trials comparing right unilateral (RUL) and BLECT was probably to blame for the inconsistent results, hence adding fuel to the controversy. Sackeim et al. ${ }^{2}$ also pointed out that 2.5 times seizure threshold was an 'arbitrary value' and also that the efficacy of the RUL ECT 'might be enhanced by a further increase in electrical dosage'.

Sackeim et al. ${ }^{3}$ have in fact conducted a landmark, double-blind randomised controlled trial designed to clarify the ongoing debate, and the hypothesis posited in their 1993 article. ${ }^{2}$ This trial, which included 80 depressed patients, clearly shows that RUL ECT is as effective as BL ECT. In this study RUL ECT was administered up to 500\% (5 times) above seizure threshold and compared with BLECT at 150\% (1.5 times) seizure threshold. At these levels the RUL ECT was equivalent to BLECT with response rates in the region of $65 \%$. Despite these relatively large suprathreshold levels the RUL group experienced less severe and less persistent cognitive deficits. Indeed, McCall et al. ${ }^{4}$ have con- firmed Sackeim's views and shown that there is a dose-response relationship to the antidepressant efficacy of RUL ECT, extending up to 12 times the seizure threshold. Indeed some early data indicate that at 6 times seizure threshold ('ultra high dose') RUL ECT may be effective in non-depressive psychotic disorders. ${ }^{5}$

\section{Method of stimulus titration}

Prinsloo and Pretorius' indicate in their review that for 'high-dose treatments a stimulus intensity of 2.5 times greater than seizure threshold is administered during the second and subsequent treat ments'

They fail to highlight the following points. The degree of suprathreshold stimulus administered to the patient will depend on a number of factors, critical among these being electrode placement, which is decided on by the treating clinician and consented to by the patient. If RUL ECT is to be administered, as indicated above it is critical that high doses be used. In order for such high doses to be given safely, the treating practitioner has to master stimulus dosing techniques and threshold estimations. This is highlighted by Rasmussen, ${ }^{\circ}$ who states that if unilateral ECT is to be used seizure threshold should be measured at the first session. For high-dose RUL ECT the majority of patients will need energies at 3 - 5 times seizure threshold. Rasmussen claims that 6 times threshold should be used (ultra high dose). ${ }^{\circ}$ However, should BL ECT be administered it is probably reasonable to assume, based on Sackeim's data, that 1.5 - 2.5 times threshold should be sufficient.? Clearly higher doses will precipitate neurocognitive sideeffects that will outweigh the clinical benefits.

It is a well-recognised phenomenon that the patient's seizure threshold increases during the course of ECT (see below). As such it may be necessary to repeat the stimulus titration to re-establish the patient's threshold during the course of the ECT. This exercise is critical if converting a patient from high-dose RUL ECT to any other electrode placement in order to avoid unnecessary overdose. Electrode placement conversion typically occurs if the patient has failed to respond to the RUL ECT trial and requires a course of bitemporal ECT. ${ }^{\circ}$ 
If high doses of RUL ECT are considered, it is the ECT practitioner's responsibility to ensure that the ECT machine that is to be used is capable of administering the high doses of energy required. For example, should a patient's threshold be established to be 100.8 mc energy (20\% on a Thymatron machine), high-dose RUL ECT would equate to around $302.4 \mathrm{mc}$ - $504 \mathrm{mc}(60-100 \%$ on a Thymatron machine), depending on whether 3 or 5 times seizure threshold was decided upon by the treating doctor. Clearly, should the patient's threshold increase during the course of the ECT it will soon be discovered that the machine is no longer capable of administering the doses at the levels needed to stay above threshold. This will mean that the patient receives inadequate treatments and undermines the therapeutic efficacy of the ECT. In order to overcome this situation many of the more modern ECT machines have the capacity to deliver over $1000 \mathrm{mc}$ (up to $200 \%$ on a Thymatron machine). While not yet available in the USA, these machines are available here in South Africa and in other countries such as Australia. It is obvious that a great deal of harm can befall the patient should such high doses be used inappropriately by a practitioner who has not had extensive training in the use of these machines.

On the other hand, there are sufficient data to show clearly that low-dose RUL ECT is clinically useless and should be abandoned.' Low-dose RUL ECT would be considered to be at levels at or near threshold and possibly up to as high as 2.5 times threshold, because even at these levels the response rate of RUL ECT is unacceptably low. ${ }^{2}$ It is therefore critical that should RUL ECT be considered, the correct equipment has to be available and the treating clinician must be appropriately trained.

\section{Practical guidelines for administering ECT}

For the arguments above to make any sense at all to the ECT practitioner, he or she would need to be well versed in the techniques of stimulus dosing, threshold estimation and UL ECT. Unfortunately this is certainly not the case here in South Africa. Given the absence of recognised ECT guidelines, training requirements and privileging rights* in this country, and the poor training in ECT that the average psychiatric registrar receives, it is not surprising that far from forming the basis of routine 'best clinical practice' these ECT techniques are virtually unknown in most local centres. This situation is unfortunately not unique to the South African context: similar problems have been documented in both the UK and the

*The right and privilege to perform this procedure in a certified and audited ECT centre - in orde to retain this right, the psychiatrist has to able to prove to a board of auditors that he sucessfully per forms a certain number of ECT procedures per year, and follows the local medical authorities recognised ECT guidelines at all times.
USA. A recent editorial highlighted that ECT in the UK is a 'neglected service' with widespread variations in practice despite the fact that very clear Royal College ECT guidelines exist. Indeed, clinical audits in the UK indicate that these guidelines are often not adhered to. ${ }^{8}$ Hermann et al. have also highlighted some of the problems with ECT service delivery in the USA. ${ }^{9}$

I myself had to receive training in these techniques in Australia before I could implement them in our local ECT unit, and I respectfully refer practitioners who would like further information about the techniques of stimulus dosing and seizure threshold estimations to some excellent articles that should help clarify the situation further. ${ }^{10,11}$

\section{Seizure length, frequency and course of admin- istration}

Prinsloo and Pretorius' quote a 1982 article when they state that 'a seizure of at least 25 seconds' duration is needed to be effective'.

There are plenty of controlled data available today showing that seizure duration', when taken in isolation, is a poor indicator of 'seizure efficacy'. Indeed, this parameter differs in various research articles, ranging from 20 to 25 seconds of motor activity, and 25 to 30 seconds of electro-encephalographic activity. ${ }^{3,4}$ In other studies these parameters were changed as the course of ECT progressed to accommodate the inevitable decrease in seizure duration that occurs with prolonged treatment with ECT. ${ }^{2}$ Some authors call these durational criteria 'conservative'. ${ }^{12}$

It is critical to realise that in the research studies that reviewed lowdose (threshold) levels of ECT, response rates were as low as $17 \%$, despite the fact that seizure durations were 'adequate' las defined above), especially if RUL ECT data are taken into account. ${ }^{2 \cdot 4}$ This indicates that seizure duration in isolation is not indicative of 'seizure efficacy'. The duration of a seizure needs to be interpreted in the light of the other treatment parameters such as electrode placement, number of previous treatments, and of course seizure threshold. Sackeim et al. ${ }^{13}$ have shown that seizure thresholds can increase enormously during a course of ECT, up to almost $90 \%$ for BL ECT and to a more modest $40 \%$ for UL ECT. This phenomenon invariably means that during the course of ECT treatment the patient's seizure duration starts to diminish until the 20 - 25-second cut-off is reached. This is because the patient's seizure threshold is increasing and gradually approaching the current levels of ECT 'dose'. At this point the treating physician increases the 'dose' in the hope of achieving a longer seizure, in 
the misguided notion that the longer the seizure, the better it is for the patient. What is in effect happening is that the dose is readjusted upwards, to be in line once again with the suprathreshold levels (1 1.5 - 2.5 times threshold for BL ECT and 3 - 6 times threshold for ULECT) we now know to be critical to clinical efficacy of the seizure. The precise minimum duration needed for a clinically effective seizure, at adequate ECT 'dose' levels above threshold, is currently not known.

\section{Conclusion}

Stimulus dosing, threshold estimations and high-dose RUL ECT are but a few of the significant technical advances that have been made in the field over the past two decades. The increasing complexities of ECT make the importance of continuous training and professional development in this discipline absolutely critical. As the science and technology of ECT continue to advance, colleagues in training and those who continue to provide ECT as a treatment option to their patients will need increasing amounts of training and supervision. Given the controversial nature of ECT as a treatment modality, and the ever-increasing risks of litigation, it is incumbent upon South African psychiatry to institute measures to control, monitor and audit the quality of ECT practised across the land, as is the current norm in First-World countries. The South

A response by Prinsloo and Pretorius arrived just too late for publication, and will appear in the next issue.
African College of Psychiatry together with other key role players should strongly consider the publication of ECT practice guidelines if we are to maintain safe and effective local ECT practices.

\section{Jose Segal}

Electroconvulsive Therapy Unit

Tara Hospital, The H Moross Centre Johannesburg

1. Prinsloo S, Pretorius PJ. Electroconvulsive therapy and its use in modern-day psychiatry. South African Journal of Psychiatry 2004; 10: 38-44.

2. Sackeim HA, Prudic J, Devanand DP, et al. Effects of stimulus intensity and electrode place ment on the efficacy and cognitive effects of electroconvulsive therapy. N Engl J Med 1993; 328: $839-846$

3. Sackeim HA, Prudic J, Devanand DP, et al. A prospective randomised double-blind comparison of bilateral and right unilateral electroconvulsive therapy at different stimulus intensities. Arch Gen Psychiatry 2000; 57: 425-434.

4. Mc Call WW, Reboussin DM, Weiner RD, et al. Titrated moderately suprathreshold versus high dose right unilateral electroconvulsive therapy. Arch Gen Psychiatry 2000; 57: 438-444.

5. Little JD, Munday J, Martin A. Right unilateral ECT at six times seizure threshold: Is it effective in the psychoses? J ECT 2003; 3: 158-163.

6. Rasmussen KG. Clinical applications of recent research on electroconvulsive therapy. Bull Menninger Clin 2003; 67: 18-31

7. Sackeim HA, Decina P, Portnoy S, et al. Effects of electrode placement on the efficacy of lowdose ECT. Am J Psychiatry 1987; 144: 1449-1445

8. Carney S, Geddes J. Electroconvulsive therapy (Editorial). BMJ 2003; 326: 1343-1344

9. Hermann RC, Dorwart MD, Hoover CW, et al. Variation in ECT use in the United States. Am J Psychiatry 1995; 152: 869-875.

10. Frukacz A, Mitchell P. Fitting the treatment to the patient: Recent advances in the practice of electroconvulsive therapy. Aust N Z J Psychiatry 1995; 29: 484-491.

11. Beale MD. Stimulus dosing methods in electroconvulsive therapy. Psychiatric Annals 1998; 28: $510-512$

12. Prudic J, Haskett RF, Mulsant B, et al. Resistance to antidepressant medications and short-term clinical response to ECT. Am J Psychiatry 1996; 153: 985-992.

13. Sackeim H, Decina P, Prohovnik I, et al. Seizure threshold in electroconvulsive therapy: Effects of age, sex, electrode placement and number of treatments. Arch Gen Psychiatry 1987; 44: 355-360. 